\title{
Infrared Spectroscopic and Scanning Electron Microscopy Study of Ibuprofen Loading onto the Molecular Sieve Mesoporous Silica SBA-15 Material
}

\author{
MARIA ULFA ${ }^{1}$ and DIDIK PRASETYOKO \\ ${ }^{1}$ Chemistry Education Study Program, Faculty of Teacher Training and Education Sebelas Maret \\ University, Jl. Ir. Sutami 36A, Surakarta 57126, Central Java Indonesia. \\ ${ }^{2}$ Department of Chemistry, Faculty of Mathematics and Natural Sciences, Institute of Technology \\ Sepuluh Nopember, Jl Keputih, Surabaya, 61115, East Java Indonesia. \\ ${ }^{*}$ Corresponding author E-mail: ulfa.maria2015@gmail.com \\ http://dx.doi.org/10.13005/ojc/340554
}

(Received: May 29, 2018; Accepted: August 04, 2018)

\begin{abstract}
The SBA- 15 was studied for the first time for ibuprofen loading as anti inflamantory molecule model. The SBA-15 was synthesized from Pluronic P123 as the structure-directing agent and TEOS as the silica source. The framework capacity and structural change of the SBA-15 was investigated by varying the amount loading of ibuprofen inflamantory molecule. The structural properties of the synthesized samples after loading treatment was analyzed by FTIR, SEM and EDX. The decreasing intensity of the SBA-15 as increasing ibuprofen loading by FTIR measurement demonstrated that a big part of mesoporous pipe has been covered by ibuprofen molecule. Spectra of FTIR also show that increasing ibuprofen molecule was decrease the number of silanol group of the SBA-15 but increase carbonyl and hydroxyl group. The SEM image show not only the average widht of the micropencil-like structure of SBA-15 close to the 1-2 mu and lenght of pipe close to the $30 \mathrm{~m} \mu$ but also white coral-like structure from ibuprofen excess. The ibuprofen molecule in one part were obtained homogeneously dispersed within the pore structure of the SBA-15 but in the other part agglomerated without uniformity. The elemental analysis by EDAX showed that the sample containing silica, oxygen, carbon and hydrogen that changed gradually as varying ibuprofen loading. The result show that the structure of SBA-15 has been stable at the given condition which is afforded a maximum loading ibuprofen up to $10 \mathrm{~mol}$ at room temperature. The slow release of ibuprofen/SBA-15 is ascribed to its unique SBA-15 structure which is achieved optimum release up to $67 \%$ within 60 minutes.
\end{abstract}

Keywords: Loading, SBA-15, Lbuprofen, Drug delivery system, Release, Structure.

\section{INTRODUCTION}

Many industrial fields have applied using mesoporous material due to their unique characteristics such as structure uniformity, large space of pore, high surface area, inert and high thermal stability ${ }^{1}$. The structure of mesoporous material has developed by numerous researcher

This is an Open Access article licensed under a Creative Commons Attribution-Non Commercial-Share Alike 4.0 International License (https://creativecommons.org/licenses/by-nc-sa/4.0/), which permits unrestricted Non Commercial use, distribution and reproduction in any medium, provided the original work is properly cited. 
to produce a much variation of ordered material species such as MCM-41, MCM-48, SBA-2, SBA15, KIT, FDU, CMK-1, CMK-3, and CMK-5². One of the mesoporous silica family which have shown high performance in many application is SBA-15. The mesoporous silica SBA-15 (Santa Barbara Amorphous) synthesized for the first time by the research group at Santa Barbara University have the tunable pore, periodic honeycomb structure, and very high surface area ${ }^{3}$. Many application of mesoporous silica material have shown great performance as catalyst, adsorbent, electrics spare part, medical material, and sensor ${ }^{4}$. Today, mesoporous silica have involved in drug delivery system as the superior carrier. However, the performance of mesoporous silica SBA-15 in drug delivery has been rarely studied.

Ibuprofen, which has chemical formula as 2-(4-(2-Methylpropyl)phenyl)propanoic acid, has been used widely not only as pain removal medicine but also inflammation in medical part by decreasing prostaglandin work $^{5}$. In non-steroidal anti-inflammatory drug (NSAID) classification, ibuprofen have maximum dosage lower than $1.2 \mathrm{~g}$ per day due to the high gastrointestinal risk 6 . For this reason, ibuprofen consumption has could minimize the gastrointestinal effect by slow rate release mode. The previous research has been reported these term by capsule, complexes, film, and dispersion of solid state. However, the relation of ibuprofen release performance using uniform structure carriers in a solid-state still not clearly understood due to the rare reportation on it. The best way to know this relation is varying dosage of ibuprofen in experimental work using the mesoporous carrier.

In the of our knowledge, the effect of different loading concentration in ibuprofen release performance using SBA-15 as the carrier has not investigated yet before. In present work, mesoporous silica SBA-15 was synthesized by pluronic-P123 and source of silica as elsewhere reportation ${ }^{7}$. Then, the loading performance of was investigated by ibuprofen impregnation onto SBA-15 varying from 3-14 mol. The textural structure of SBA-15 after loading preparation was characterized by FTIR, SEM, and EDX. At the end of our work, the release performance using simulated body fluid solution was studied by UV-Vis measurement.

\section{EXPERIMENT}

\section{Materials}

In this work, the nonionic triblock copolymer poly(ethylene oxide)-poly(propylene oxide)poly(ethylene oxide) surfactant where named as Pluronic P123 with molecular weight up to 5800 have been used as a soft template which is purchased from Sigma-Aldrich. The tetraethyl orthosilicate (TEOS) has been used as silica source which purchased from Sigma. Hydrochloric acid, Ibuprofen, and $n$-Hexane were purchased from Merck \& Co. Another chemical including the chemical to prepare solution body fluid was purchased from Merck \& Co. and Sigma-Aldrich.

\section{Synthesis of mesoporous silica SBA-15}

The mesoporous silica SBA-15 sample synthesized based on Nejad et al., procedure with small part modification ${ }^{8}$. The pluronic p123 was mixed in hydrochloric acid solution to obtain the P123 solution. The tetraethyl orthosilicate drops slowly into P123 solution under stirring 150 rpm for 24 hours. The ratio of pluronic p123: tetraethyl orthosilicate: the hydrochloric acid solution is 1:5:30 (w/w/v). The mixture was transferred into steel container and aged at $10^{\circ} \mathrm{C}$ for 24 as a hydrothermal step. The white composite separating with vacuum apparatus, washing with distilled water and drying in the oven at $10^{\circ} \mathrm{C}$ for 24 hours. Then, for removing the p123 part, the grey-white powder was calcined at $550^{\circ} \mathrm{C}$ in air for $24 \mathrm{~h}$, the resulting sample then kept on desiccator until next application process.

\section{Loading and release ibuprofen at in vitro system}

The different weights of Ibuprofen were mixed with hexane to obtain ibuprofen solution at concentration 4,10 and $13 \mathrm{~mol}$. In the close batch, the ibuprofen solution was infiltrated onto mesoporous silica SBA-15 followed by homogenization at room temperature for 24 and kept from evaporation. The white material was filtered by Whatman filter paper no 41 then dried at room temperature for 24 hours. The resulted sample labeled as $x$-Ibuprofen/SBA-15, where $x$ is the concentration of ibuprofen. The coin plate-like of the sample was prepared by $\mathrm{x}$-Ibuprofen/ SBA-15 press with the pelleting system at 3 Torr. The coin plate-like of Ibuprofen/SBA-15 sample was mixed into simulated body fluid solution at a ratio $(3: 5 \mathrm{w} / \mathrm{v})$ then followed by stirring at $37^{\circ} \mathrm{C}, 150 \mathrm{rpm}$ for 10 minute. After this process, the filtrate was token by 
syringe in interval 3 minute. The filtrate concentration then measured by UV-Vis spectroscopy at $\lambda=272$ $\mathrm{nm}$. Ibuprofen position in ibuprofen/SBA-15 sample was characterized by SEM, EDS, and FTIR.

\section{Characterization}

All sample were characterized respectively by scanning electron microscopy (JEOL, JSM-
6700F, $100 \mathrm{kV}$ ), Fourier transform infrared (FT-IR) (Shimadzu, FTIR036) and EDS EDX (Energy Dispersive X-ray Analysis). Scanning electron microscopy was used as structure and morphology examiner. Fourier transform infrared spectra obtained by coin disk method with $\mathrm{KBr}$ recorded at wavenumber $400-4500 \mathrm{~cm}^{-1}$. Elemental content Ibuprofen/SBA-15 samples were measured by EDX.

Table 1: Mapping element of mesoporous silica SBA-15 at different loading concentration by EDS

\begin{tabular}{lccccc}
\hline Sample & \multicolumn{5}{c}{ Weight \% } \\
& $\mathrm{C}$ & $\mathrm{O}$ & $\mathrm{Si}$ & $\mathrm{Mg}$ & others \\
\hline 3-ibuprofen/SBA-15 & 16.11 & 44.12 & 39.77 & - & - \\
10-ibuprofen/SBA-15 & 20.04 & 45.08 & 33.91 & 0.75 & 0.21 \\
14-ibuprofen/SBA-15 & 24.84 & 47.84 & 26.75 & 0.56 & - \\
Pure SBA-15 & - & 48.70 & 51.30 & - & - \\
\hline
\end{tabular}

\section{RESULT AND DISCUSSION}

Figure 1 shows the spectra of Ibuprofen/ SBA-15 at different ibuprofen concentration. The incorporation of ibuprofen onto mesoporous silica SBA-15 clearly observed by FTIR spectroscopy. The -C-H stretching vibrations from ibuprofen molecule observed in infrared peaks at 2925, 540 and 424 $\mathrm{cm}^{-1}$. the ibuprofen position not only shows $-\mathrm{C}-\mathrm{H}$ but also $\mathrm{C}=\mathrm{O}$ stretching vibrations at $625 \mathrm{~cm}^{-1}$. While the peaks at $1535 \mathrm{~cm}^{-1}$ and $1620 \mathrm{~cm}^{-1}$ corresponds to $\mathrm{Si}-\mathrm{O}-\mathrm{Si}$ (silanol group) which is also strongly observed at the peaks $3440 \mathrm{~cm}^{-1}$ as $-\mathrm{Si}-\mathrm{OH}$ group. In the term of ibuprofen and silica interaction, the anchor between mesoporous silica SBA-15 surface and the center of ibuprofen molecule was successfully acted by hydrogen of the secondary hydrogen of $\mathrm{C}-\mathrm{H}$ as base covalent bond. The backbone and ring vibration observed in the other of main peaks.

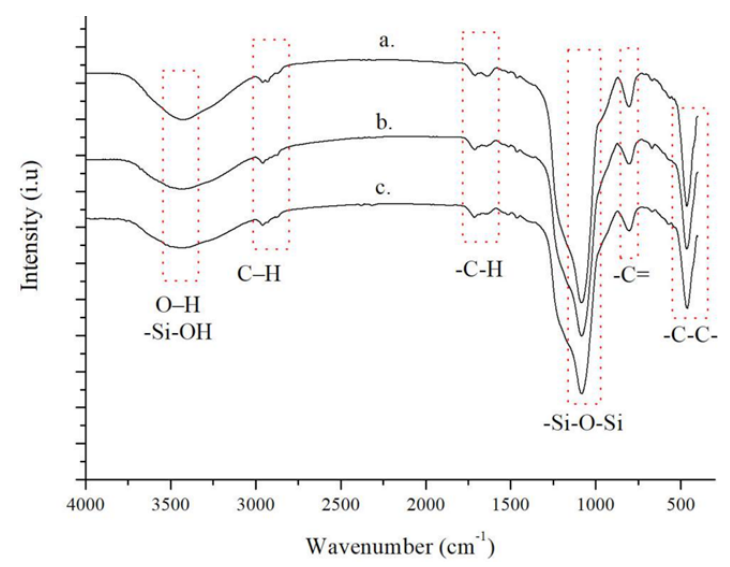

Fig. 1. IR spectra sample of 3-ibuprofen/SBA-15 (a), 10ibuprofen/SBA-15 (b), and 14-ibuprofen/SBA-15 (c)
Spectra FTIR Fig.1.a showed that increasing ibuprofen concentration following with decreasing peak of $\mathrm{C}=\mathrm{O}$ due to the inaccessible SBA-15 pore for a big number of ibuprofen. The molecule of ibuprofen size is closely $1-3 \mathrm{~nm}$ contain 13 carbon, 18 hydrogen, and 2 oxygen ${ }^{9}$. The difficulties of ibuprofen infiltration onto SBA-15 affected by blocking pore due to the high ibuprofen concentration impact. After small part of ibuprofen covered the main pore of SBA-15, another ibuprofen molecule could not be deposited directly by loading on the inner SBA-15 surface. This excess of ibuprofen leaching out after washing process then effected in decreasing carbonyl peak in spectra. In the other side, the increasing ibuprofen concentration followed with broadening peak of silanol side at $3400 \mathrm{~cm}^{-1}$ and $1600 \mathrm{~cm}^{-1}$. The broadening phenomenon clearly observed at Fig. 1c which corresponds to small coverage of SBA-15 surface due to the high loading. However, all the spectra showed that structure of mesoporous silica SBA-15 still stable without significant destruction. In addition, the ibuprofen molecule was successfully loaded onto SBA-15 in given ibuprofen concentration.

Figure 2. shows the SEM of the ibuprofen/ SBA-15 sample at different loading concentration. As shown in Fig. 2a at rectangle red circle, the ibuprofen/ SBA-15 sample present monopencil-like structure with an average length of around 20-50 $\mu$. The group of ibuprofen which could not deposit onto inner pore of SBA-15 was observed clearly in SEM image as 
the white coral-like structure. The increasing loading concentration followed with increasing the spot of white coral-like in the whole image. It probably appears because ibuprofen overlaps each other to form the stack of coral-like. It can be good evidence to strongly support spectra FTIR result. Image from SEM describes the stability of mesoporous SBA-15 which is observed as a two-dimensional monopencil-like structure in large of an image area. The mesoporous SBA-15 also measured with the same average length which indicates the high uniformity of structural material after loading step.

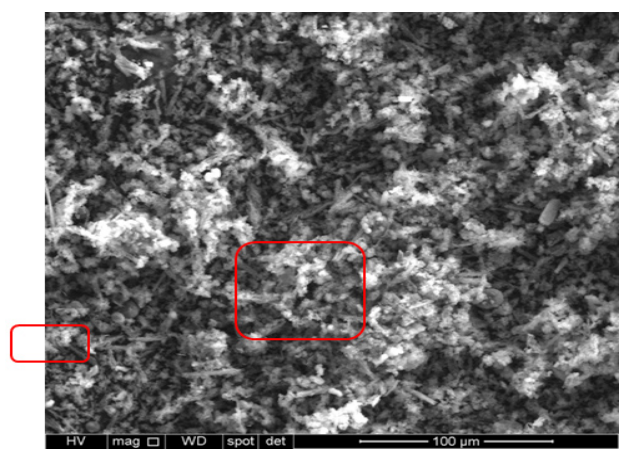

(a)

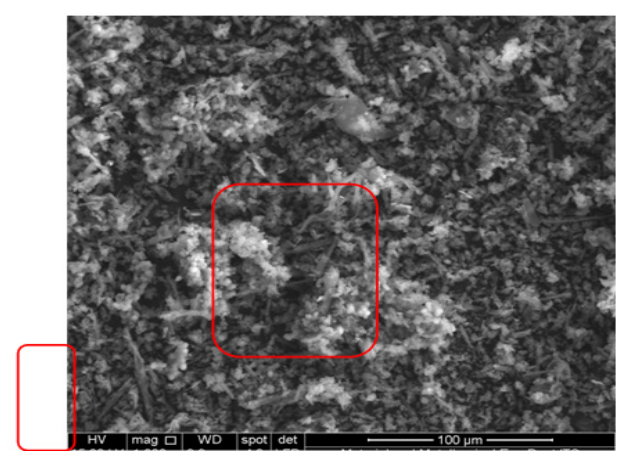

(b)

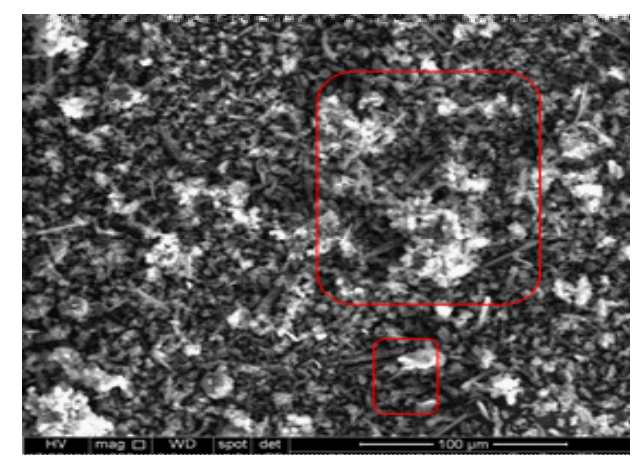

(c)

Fig. 2. SEM image sample of 3-ibuprofen/SBA-15 (a), 10-ibuprofen/SBA-15 (b), and 14-ibuprofen/SBA-15 (c)
Other information of ibuprofen/SBA-15 SEM shows that ibuprofen has been successfully incorporated on the inner surface of SBA-15. The 10-ibuprofen/SBA-15 sample (Fig. 3a) show highest monopencil-like than other sample probably as the optimum loading onto SBA-15. It is logic because the competition between each ibuprofen molecule at low level in lower loading. The bulk space available in large area affected in increasing blocking pore. So, the failed ibuprofen covered the outer space and observed as the spot of white coral-like structure in SEM image. In another way, 10-ibuprofen/SBA15 sample shows the monopencil-like structure with small part of white coral-like as the high competition effect from ibuprofen. The increasing ibuprofen loading concentration has not destructed significantly SBA-15 is reasonable because, in all loading concentration, monopencil-like structure observed dominantly in SEM area. The numerous monopencil-like structure clearly observed indicate that ibuprofen not only infiltrate at inner pore but also covered the outer surface with slim layer. All the SEM image The ibuprofen loading process have not affected the damage of the mother pipe of SBA-15 due to the smooth coverage step during impregnation. The SEM result give information about the incorporation ibuprofen molecule onto SBA-15 which is described also by EDX analysis

Figure 3 shows mapping element of pure SBA-15 and ibuprofen/SBA-15 sample by energydispersive X-ray (EDX) spectra. In all sample, the three main peaks in ibuprofen/SBA-15 sample were observed as carbon, silica and oxygen. This result indicating that ibuprofen/SBA-15 sample contain C-Si-O element. The pure SBA-15 contain $47 \%$ silica and $53 \%$ oxygen. The peaks of carbon and oxygen detected in EDX spectra due to the presence of ibuprofen. The interesting result appears by each sample where the carbon content enhances gradually from 16,20 to $24 \%$ at sample 3,10 and 14 ibuprofen/SBA-15. This question can be answered using optimizing package view. Ryoo reported that every space of ordered mesoporous material has the maximum capacity to filled by some molecule ${ }^{10}$. In optimizing package, SBA-15 can filled by ibuprofen at certain interval. The whole ibuprofen could not 
deposited completely due the maximum package of SBA-15. It can be the next evidence after FTIR and SEM result which is describe that ibuprofen has been successfully infiltrated and covered the surface of SBA-15. The peaks of Si were detected as SBA-15 in the EDX spectra. The presence of $\mathrm{Mg}$ and $\mathrm{Al}$ in the EDS spectra indicating that small impurities involves during preparation but not significantly change the whole sample.

The increasing ibuprofen loading followed with the amount of carbon as second major element after hydrogen and oxygen. An interesting view, the percentage of oxygen closely same in all sample. It can deeply understand if we presume that all oxygen coming from SBA-15 and ibuprofen. This estimation appears from the FTIR result which is a functional group of $-\mathrm{C}-\mathrm{H}$ as an anchor between ibuprofen and SBA-15. After anchoring step, a small part of ibuprofen oxygen covered the oxygen of SBA-15 then leach out during washing treatment. In the second assumption, it is impossible to leach out ibuprofen oxygen from the sample because the bonding of $\mathrm{C}=\mathrm{O}$ stronger than $\mathrm{Si}-\mathrm{OH}$ which ibuprofen granulation is still observed as white coral-like structure after loading treatment. This phenomenon related to the decreasing silica content with increasing ibuprofen loading. In incorporation process, SBA-15 have numerous pore as some vacancies for ibuprofen. The vacancies partially filled by ibuprofen and in another word, the position of silica from SBA-15 replace with carbon from ibuprofen with oxygen available in the similar amount to keep material stability.

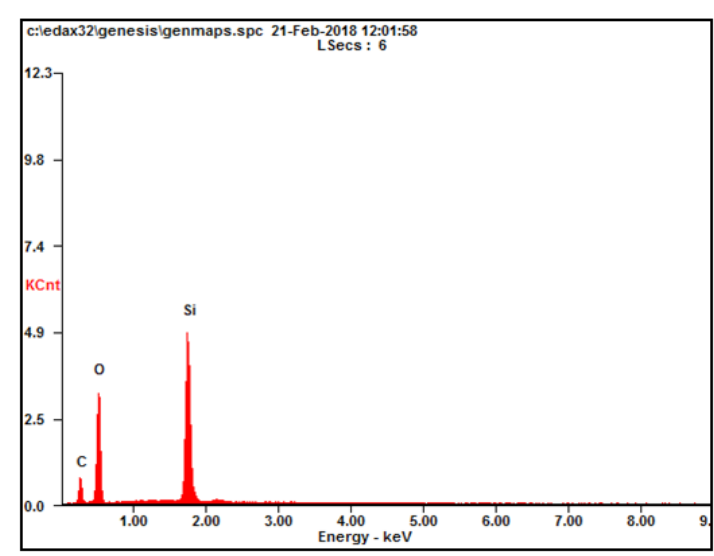

(a)

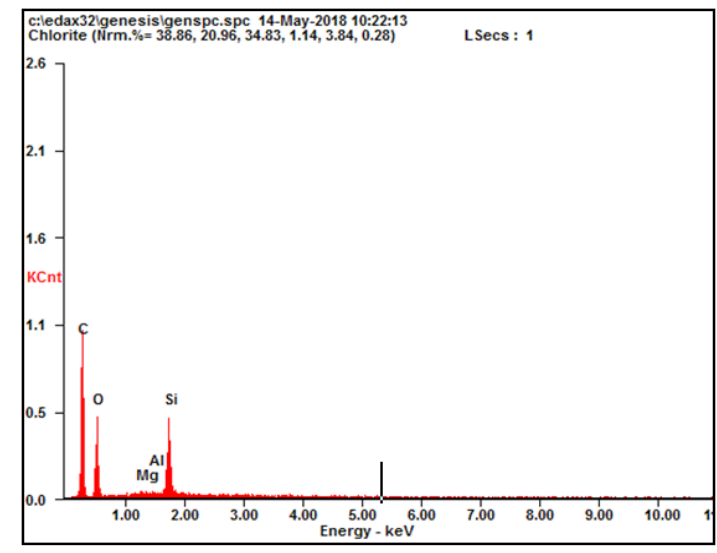

(b)

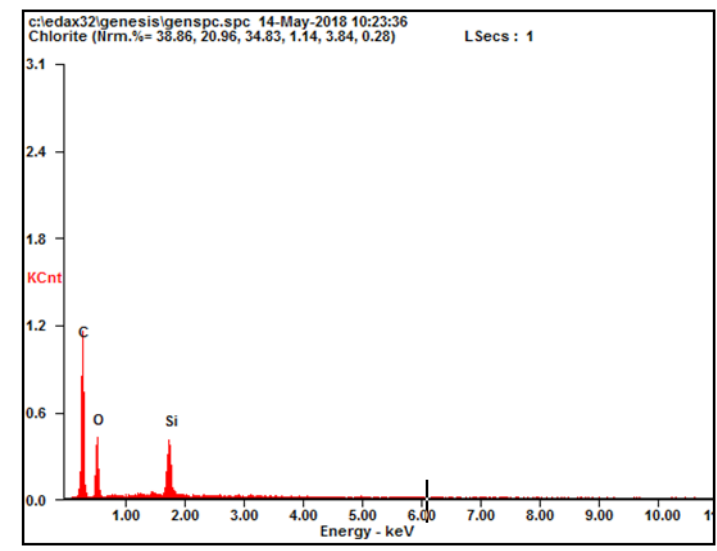

(c)

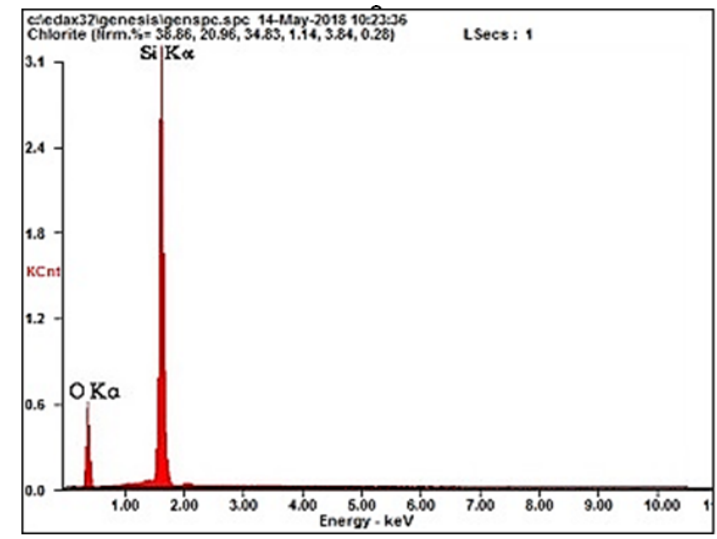

(d)

Fig. 3. Elemental compositon samples of 3-ibuprofen/SBA15 (a), 10-ibuprofen/SBA-15 (b), 14-ibuprofen/SBA-15 (c) and pure SBA-15 (d)

Figure 4 shows the trend of ibuprofen release from samples at simulated body fluid temperature. As can be seen, ibuprofen release from 10-ibuprofen/SBA-15 sample was $66 \%$ but 14 ibuprofen/SBA-15 sample was released only $45 \%$ 
after 60 minute. It is probably happened by slow release after ibuprofen deposited in an inner pore of SBA-15. Ibuprofen molecule in high loading sample difficult to leach out from SBA-15 pore due to the reblocking pore and high competition ibuprofen each other. The reblocking probably have been caused not only by high affinity of silanol from SBA-15 and carbonyl from ibuprofen via hydrogen bonding of $\mathrm{C}-\mathrm{H}$ but also the across position of ibuprofen was appopriate with mouth pore size of SBA-15. Similar with loading phenomenon, a number of ibuprofen molecules were trapped at the pore mouth which is affected in slow release rate.

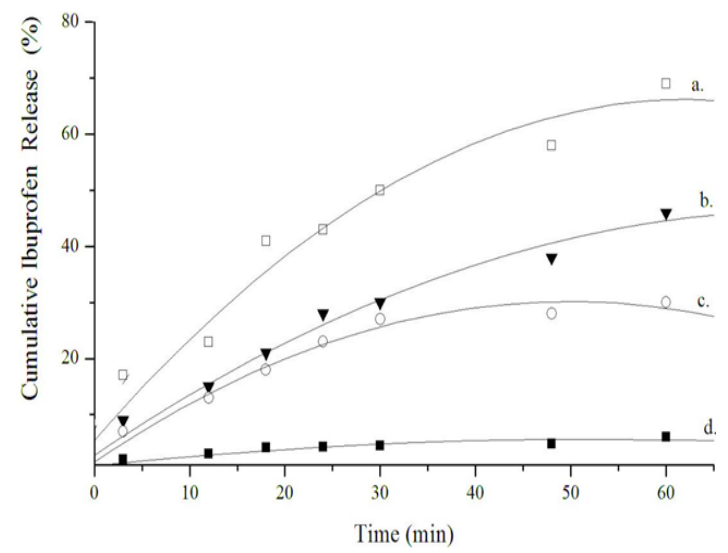

Fig. 4. Release trend of ibuprofen from samples of 10ibuprofen/SBA-15 (a), 14-ibuprofen/SBA-15 (b),3-ibuprofen/ SBA-15 (c) and ibuprofen (d)

In whole body system, trapping effect in drug release give a big advantage for prostaglandin response decreasing gradually. As we know, prostaglandin limitation work could be served enough time for the stomach to adsorb ibuprofen as drug $^{11}$. If ibuprofen consumption using mesoporous silica SBA-15 not only could control the ibuprofen release but also minimize gastrointestinal risk on the in vivo model. As seen from the resulting research, slow release of mesoporous silica SBA-15 sample could be the best reference to drug delivery carrier with lower gastrointestinal effect.

\section{CONCLUSION}

A mesoporous silica SBA-15 was successfully synthesized using impregnation method. Incorporation ibuprofen onto mesoporous silica SBA-15 material was completely happened by simple loading preparation. The ibuprofen/SBA-15 composite obtained possessed a micro pencil-like structure with white coral-like from ibuprofen excess structure. The whole result shows that the SBA-15 microstructure had not greatly damaged by the loading process. The big potential of SBA-15 has shown by release performance test using simulated body flow solution as an in-vitro system. The end of our research concludes that the optimum sample loading had been minimized stomach problem in ibuprofen consumption.

\section{ACKNOWLEDGMENT}

Our research was financially supported by the Directorate General of Higher Education Indonesia under the Post Doctoral Grant 2018 (grant number 474/UN27.21/PP/2018)

\section{REFERENCES}

1. Yang, J., Zhai, Y., Deng, Y., Gu, D., Li, Q., Wu, Q., Zhao, D. Journal of Colloid and Interface Science., 2010, 342(2), 579-585.

2. Guo, R., Guo, J., Yu, F., \& Gang, D. D. Microporous and Mesoporous Materials., 2013, 175, 141-146.

3. Fan, C. Y. J., Zhang, B. T. F., \& Zhao, D. Carbon., 2003, 20, 45-48.

4. Xiang, L., Royer, S., Zhang, H., Tatibouët, J. M., Barrault, J., \& Valange, S. Journal of Hazardous Materials., 2009, 172(2-3), 1175-1184.

5. Zhou, Q., Shi, L., Marinaro, W., Lu, Q., \& Calvin, C. Powder Technology., 2013, 249, 290-296.

6. To, D., \& Davé, R. N. Powder Technology.,
2016, 290, 102-113.

7. Chandrasekar, G., Son, W. J., \& Ahn, W. S. Journal of Porous Materials., 2009, 22-33.

8. Farzin Nejad, N., Shams, E., Amini, M. K., \& Bennett, J. C. Microporous and Mesoporous Materials., 2013. 239-246.

9. Fröhlich, A. C., Ocampo-pérez, R., Diaz-blancas, V., Salau, N. P. G., \& Dotto, G. L. Chemical Engineering Journal., 2018, 341, 65-74.

10. Khodakov, A. Y., Zholobenko, V. L., Bechara, R., \& Durand, D. Microporous and Mesoporous Materials., 2005, 79,1-3, 29-39.

11. Zhang, L., Cheng, H., Zheng, C., Dong, F., Man, S., \& Dai, Y. Journal of Drug Delivery Science and Technology., 2016, 31, 101-107. 